\title{
Development of a Conceptual Model of Resilience in New Graduate Nurses
}

\author{
Lei Lyu ${ }^{1, \text { * }}$, Lingyan $\mathrm{Xu}^{1}$, Guohong $\mathrm{Li}^{2, \text { * }}$ \\ ${ }^{1}$ Department of Emergency, Women's Hospital School of Medicine Zhejiang University, Hangzhou, China \\ ${ }^{2}$ Department of Nursing, Zhongda Hospital Affiliated to Southeast University, Nanjing, China
}

Email address:

lyulei azju.edu.cn (Lei Lyu), 1325118124@qq.com (Guohong Li)

${ }^{*}$ Corresponding author

\section{To cite this article:}

Lei Lyu, Lingyan Xu, Guohong Li. Development of a Conceptual Model of Resilience in New Graduate Nurses. American Journal of Nursing Science. Vol. 7, No. 4, 2018, pp. 159-164. doi: 10.11648/j.ajns.20180704.18

Received: July 6, 2018; Accepted: July 16, 2018; Published: August 15, 2018

\begin{abstract}
To explore experiences of stress-tolerance among new graduate nurses and to develop a conceptual model of resilience. The formation rules of the conceptual model can be found. It's necessary to examine resilience positively affecting new nurses at different stages of transition period. Using a grounded theory approach, 12 outstanding new nurses aged 20-26 years whose working time was more than 12 months but less than 24 months were interviewed. Dynamic adjustment process was the core category of the conceptual model of resilience, including three sub-processes, (focus on self-survival, clinical environment, and patient), fifteen major categories and thirty sub-categories. The process consisted of three main phases: 'Paying Attention to Self-Survival', 'Caring for Clinical Situations', and 'Caring for Patients'. The feedback of head nurses can make new nurses recognize their strengths and weaknesses, and feel the warmth from the leaders, which is favorable to the improvement of new nurses' working ability. Flexible scheduling likely creates a good working atmosphere and maintains the mental health of new nurses, which can make them learned much skills from the senior nurses, and help them smoothly adapt to the work environment. The personalized instructions and career-planning are crucial to strengthen the resilience of new nurses. External supports mean that the nurses can obtain the supports and help from a partner, family or friends, which become a key factor to affect the resilience of the nurse. Self-management, right values, independent learning, a firm belief and other personal factors are important internal factors for promoting the development of nurses' resilience. This conceptual model can be used to understand connotation of resilience in new graduate nurses, and to facilitate nurses' role transition. It's likely to provide reference for nursing managers to formulate relevant strategies, greatly reducing the turnover rate and alleviating the shortage of nurses in global nursing workforce.
\end{abstract}

Keywords: New Graduate Nurse, Role Transition, Resilience, Qualitative Study, Grounded Theory Approach

\section{Introduction}

Resilience, also known as toughness or resilience force, refers to the individuals that can smoothly adapt to adverse living-environment and develop some positive ability when facing with certain difficulties or threats [1]. Resilience first focused on the psychological recovery ability of children after trauma [2]. With the development of psychology, psychological resilience has gradually become a hot topic in international psychological research, and been widely used in various fields. Recent years, the study of nurses' resilience has aroused great concern and attention from the scholars, especially the research on the psychological resilience of new nurses [3]. New nurse is being in the transition period from students to clinical nurses [4], which belongs to the key period for the new nurse's career development. It not only affects the mastery of the new nurses' clinical knowledge and skills, but influences the job satisfaction and future career development plan of the new nurses. Some studies reported a high turnover rate among the new nurses [5-6], and the turnover rate of junior nurses is as high as $68.5 \%$ in China [7]. The new nurses often chooses to give up nursing profession, which causes the 
waste of nursing education resources, and affects the quality and safety of nursing, finally resulting in a permanent loss in human resources of nursing [8-10]. Therefore, it is particularly important to find positive and effective strategies to help new nurses get through the transition period. When facing the same complex work environment and challenges, the new nurses with the resilience can afford to deal with the challenges, and achieve career development during the transition period. This is greatly conducive to the stability of the nursing team. This study is to explore a conceptual model of resilience on outstanding new nurses in the transition period, and find its formation rules. It's likely to provide reference for nursing managers to formulate relevant strategies.

\section{Samples and Methods}

\subsection{Samples}

\subsubsection{Selection}

A purposive sampling method was adopted, and the new clinical nurses from Zhongda Hospital Affiliated to Southeast University were selected. Based on the definition of new nurses' transition period from Jewell [3], the understanding of outstanding new nurses are presented as follows: 1. 1 2 years in the clinical experience 2. the ability of independently performing night shift work 3 . the obtainment of thanks from patients or family members for more than 3 times 4 . the recommendation from the director and head nurse. The final inclusion criteria can be described: 1 . registered nurses 2 . the standard on the outstanding new nurses 3. clear language expression 4. voluntary minds to participate. The sample size is determined by the repeated information provided by the interviewees, and no other new topics (data saturation) are presented in data analysis.

\subsubsection{General Information}

12 new nurses (11 females, 1 male) participated with 20-27 (M=23.3, $\mathrm{SD}=2.0)$ years old. Among them, there exist nurses unmarried $(\mathrm{n}=11)$, college students $(\mathrm{n}=6)$, undergraduate $(n=4)$, masters $(n=2)$, internal medicine $(n=3)$, surgical department $(n=4)$, pediatrics $(n=1)$, intensive care unit $(n=2)$, and emergency department $(n=1)$. The study was approved by Research and Ethics Committee of S. U. (project number 2016ZDSYLL088). All interviewees were informed the content about the study, and signed an informed consent.

\subsection{Methods}

\subsubsection{Data Collection}

The interview outline was drawn up as follows: 1 . What do you feel from students to the clinical nurses? 2. What's your skills to do well in the nursing work? 3. How do you relieve the stress in the work? 4. What do you rely on to overcome the challenges in the work? 5. What's changes over a year of the clinical work? Before the interview, the permission of the ward manager was obtained, and the interviewee was contacted. The purpose of the study was introduced and the appropriate time and place for the interview was selected based on the wishes of the interviewees. The way and content of the interview can be adjusted, and the interviewer keeps the neutral attitude towards the questions over the interview. The uncertain content should be confirmed again to conform to the true experience of the interviewees. The whole process was recorded. During the interview, the interviewer listens carefully, and observes the changes of the emotion and expression of the interviewees. The time of the interview lasts 45-60 min.

\subsubsection{Data Analysis}

The first author timely completed the transcript of the data, and independently finished the data analysis with the second authors, respectively. The interviewees were named after the coding N1 N12. The software Nvivo 10 was used to analyze data, and the way of open login, relational login and core login was applied to conclude the content of the interview. In the process of the analysis, the researchers read the transcriptional text repeatedly, the data related to the elasticity were classified, the phenomenon of the elasticity were compared to sum up the concepts that can describe the phenomenon, and then recorded the related information and thinking about the code and concept. And the generalized concepts can be further categorized. Finally, the relationship among categories, or between categories and concepts were found to result in the core category.

\subsubsection{Quality Control}

The trustworthiness standard of this study followed four evaluation criteria of credibility, transferability, dependability and confirmability created by Lincoln. In terms of credibility, the leader of this study is a well-trained nursing researcher with extensive experience in clinical nursing and nursing education. And the study is designed and supervised by the leader. Considering the dependability, the collected data will be returned to the interviewees to verify the validity of the data. As for the confirmability, the participants went over the content of the translation and validated the findings of the study. The research steps, sampling methods, sample demographic characteristics, data collection and analysis process are presented to ensure its transferability.

\section{Results}

The development of resilience of new nurses can be divided into three stages: paying attention to self-survival, caring for clinical situations and caring for patients. Each process has different concepts of resilience, which can be classified into 15 main categories, 30 sub-categories, as shown in Figure 1 and Table 1. 


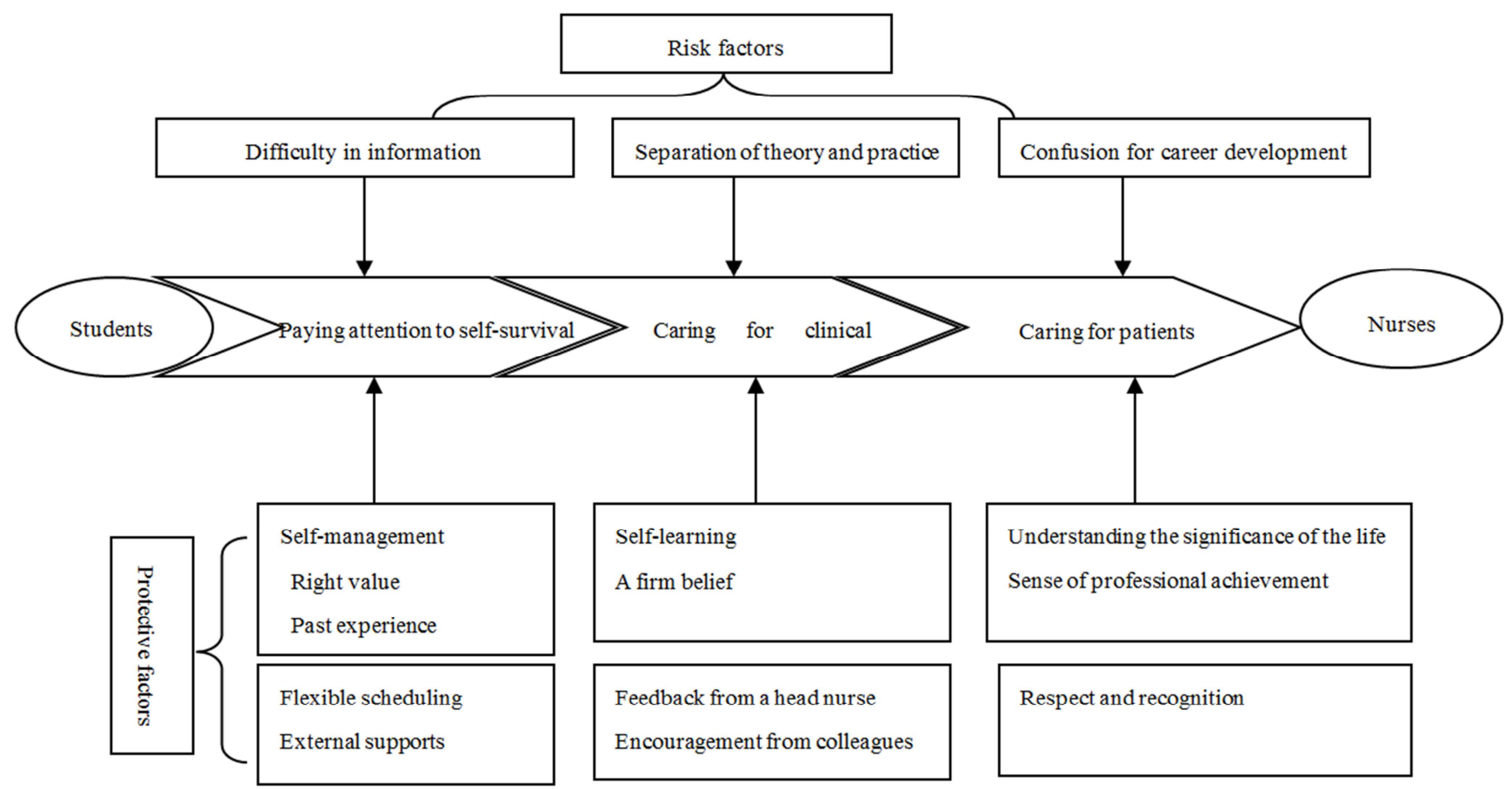

Figure 1. Development of a conceptual model of resilience in new graduate nurses.

Table 1. Three stages, fifteen major categories and thirty sub-categories.

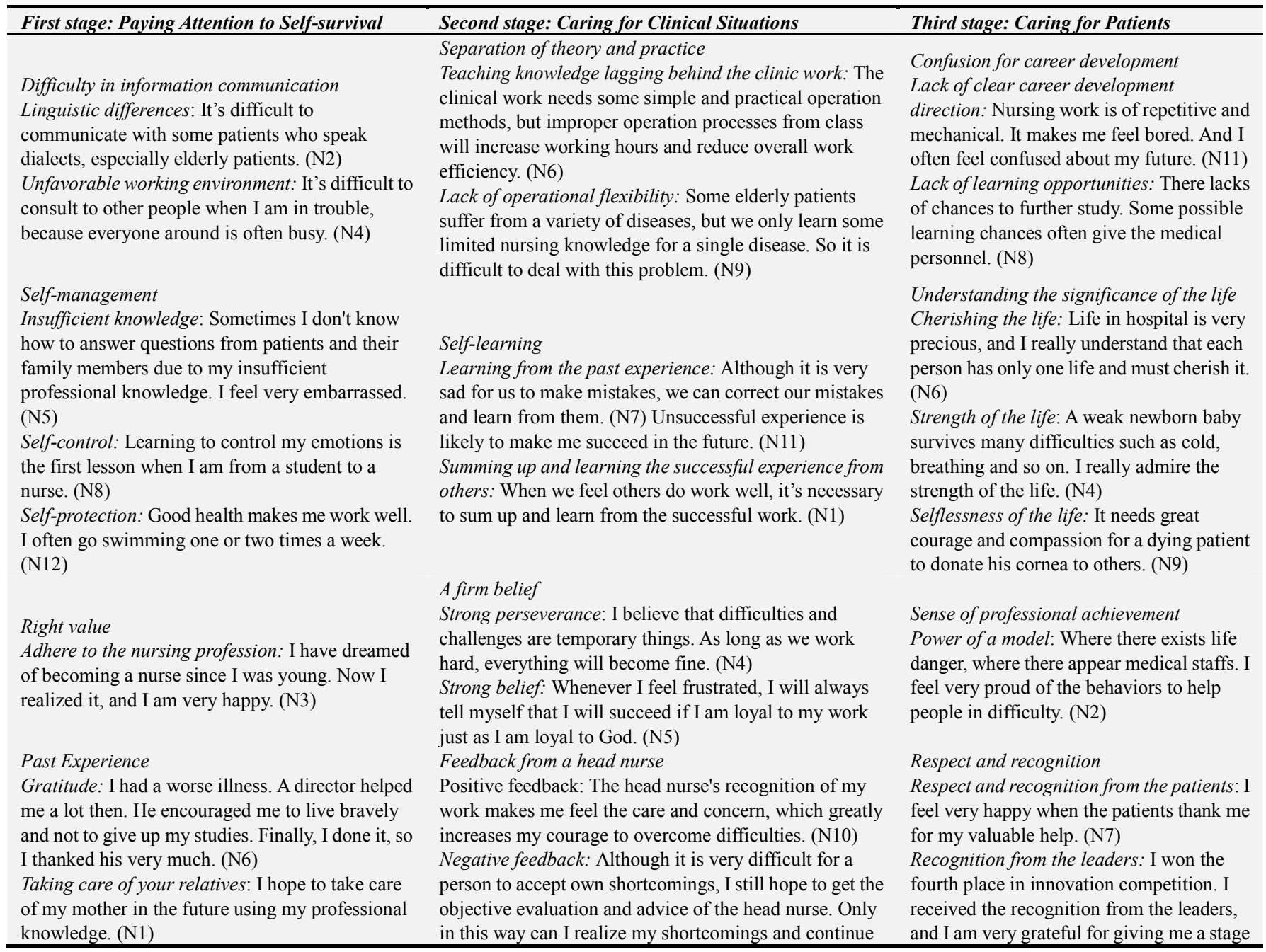




\begin{tabular}{|c|c|c|}
\hline First stage: Paying Attention to Self-survival & Second stage: Caring for Clinical Situations & Third stage: Caring for Patients \\
\hline $\begin{array}{l}\text { Flexible scheduling } \\
\text { Cooperation of nursing staffs: When I work with } \\
\text { an experienced colleague, I learn a lot from her, } \\
\text { and then I feel confident in finishing the work } \\
\text { well. (N4) } \\
\text { Relationship between ability and the patient's } \\
\text { condition: I feel worried about taking care of the } \\
\text { patients with serious illness because of my } \\
\text { inadequate professional ability. (N5) } \\
\text { External supports } \\
\text { Parent's supports: when I am not happy, my } \\
\text { parent will call me and comfort me. (N10) } \\
\text { Friends'supports: I often got help from my } \\
\text { friends by the way of mutual encouragement. } \\
\text { (N12) }\end{array}$ & $\begin{array}{l}\text { to make progress. (N9) } \\
\text { Encouragement from colleagues } \\
\text { Help from colleagues: I am very grateful to my } \\
\text { colleagues. They didn't dislike me for being a novice. } \\
\text { They always help me when I am in trouble. (N3) } \\
\text { Comfort from colleagues: Sometimes I do not do well in } \\
\text { work, and I am often accused by the patients. My } \\
\text { colleagues always comfort me and share their similar } \\
\text { experiences, which makes me feel the warmth from a big } \\
\text { family. (N12) }\end{array}$ & to show myself. (N5) \\
\hline
\end{tabular}

\section{Discussion}

\subsection{Conceptual Model of Resilience of New Nurses}

Resilience focused on the development of individual potential and exploration of psychological resources. And when facing with adversity, threats, natural disasters and other life pressure, the individual can successfully adapt to the situation, and achieve a good development. Rushton et al. [11] reported that resilience is an important predictive factor of occupation burnout, and the individuals with high resilience show a lower level of occupation burnout. The resilience can reduce the work pressure, and promote career development of the new nurses. Hart et al. [12] believed that the development of resilience can be used as an effective strategy to reduce the loss of nurses' human resources. Through the analysis of the interview data, the resilience of the new nurse shows a dynamic adjustment process, which can be summed up to three sub-processes of paying attention to self survival, caring for clinical situations and caring for patients (Figure 1). It reflects the process of resisting the pressure, adapting to the environment, and realizing the transformation of the role and the career development. Clearly, the conceptual model of resilience is consistent with the definition of psychological resilience. Blamire [13] reported that the appropriate pressure and difficulties can promote the development of resilience. The interviewees in the study show depression, confusion, and even withdrawal behavior in the transition period. This does not mean that they lack of flexibility, but experience the resilience process. This study suggests that the resilience of the new nurses is not a linear development, and each stage includes the risk and protection factors (Figure 1). That is, the interviewees constantly adjust the measurements to deal with the problems and conflicts in the workplace, further promoting the development of the resilience. Thus, this study further supports Blamire's opinion, and proves that the resilience is an interaction process of sustainable development between personal attributes and organization, interpersonal relationship and social resources [14]. Besides, the study also found that the concept of resilience of new nurses not only includes behavior to overcome the difficulties, as well as confidence, faith, spiritual encouragement from colleagues etc. Therefore, the resilience development of new nurses is conducive to change their behaviors, and improve the physical and mental health of new nurses, finally increasing the job satisfaction and reducing the turnover rate.

\subsection{Three Stages}

\subsubsection{Paying Attention to Self-Survival}

Paying attention to self-survival exist the initial stage of nurse growth, where the new nurses realize that they belong the lowest level members of the department and, only depend on themselves. However, their clinical knowledge and skills cannot meet the needs of the work, while they are afraid of loneliness, making mistakes and receiving criticism. Therefore, they are very concerned about their survival adaptability. At this stage, the new nurses generally believe that poor communication with patients, family members, or other colleagues becomes an important threat to their adaptability. At this point, the internal protective factors play a more important role in increasing the resilience, which includes self-management, right-value and past experience. Self management is divided into self-control and self-protection. Self-control refers to the control of their bad behavior to reduce the negative impact on the nursing work. And self-protection refers to adoption of the good behavior to promote their physical and mental health. External protection factors include flexible scheduling and external supports.

\subsubsection{Caring for Clinical Situations}

When new nurses are familiar with the clinical working environment and the nursing work system, they will shift their attention to improving their work ability. But at this time, they cannot skillfully distinguish the information from the clinical situation, and deal with the problems only based on the theory knowledge from the book and the stereotyped rules, causing the separation of the theory from the practice. The reflection and summary from the clinical practice is advantaged for promoting the development of the resilience of the new nurses. Besides, the feedback from nurse heads and the encouragement from the colleagues are conducive to stimulating new nurses' confidence and making them adhere 
to the nursing work, as seen in Table 1.

\subsubsection{Caring for Patients}

After more than a year work in clinical practice, the new nurses recognize the nursing difference on the different patients, so that they learn the thinking from other people's position, and gain a sense of professional accomplishment from the devotion to the nursing work. Meanwhile, the respect and recognition from other people further affirm their value. These protective factors are conducive to the development of resilience of new nurses. However, the development of the resilience of new nurses is facing the risk factor, the confusion of career development, as shown in Table 1.

\subsection{Significance of Constructing a Conceptual Model of the Resilience}

The leadership style of nursing managers makes an important effect on the resilience of new nurses [15]. The similar findings are found in this study, as follows: 1 . the feedback of head nurses can make new nurses recognize their strengths and weaknesses, and feel the warmth from the leaders, which is favorable to the improvement of new nurses' working ability. 2. flexible scheduling likely creates a good working atmosphere and maintains the mental health of new nurses. Most new nurses reflect that when facing strange people and unfamiliar circumstances at the initial work, they are unwilling to give up, but shy to ask for help from others. Flexible scheduling can make them learned much skills from the senior nurses, and help them smoothly adapt to the work environment. 3. the personalized instructions and career-planning are crucial to strengthen the resilience of new nurses. In fact, at the later stage of role transformation, the new nurses have inadequate time to learning, and no clear career-development direction, so they often feel confused about their future.

External supports mean that the nurses can obtain the supports and help from a partner, family or friends, which become a key factor to affect the resilience of the nurse [16]. The study reveals that under the huge pressure of the work, they prefer to relieve pressure or relax themselves through the Internet. Therefore, the leaders should make full use of Internet resources, and establish effective support network system to create a harmonious working-environment.

This study also finds that self-management, right values, independent learning, a firm belief and other personal factors are important internal factors for promoting the development of nurses' resilience. It is consistent with the view from Cope [17]. Positive personality quality is the source to promote the development of the resilience [18]. Therefore, nursing managers should take the individual value and needs of each new nurse into consideration, fully excavate the internal protection factors to stimulate the enthusiasm and self-worth, finally enhancing the sense of professional identity, and improving the resilience of the new nurses.

\subsection{Limitations}

First, the choice of the participants in this study only comes from the recommendation of the director and head nurses of the department. There probably exist some differences on the understanding of the outstanding new nurses. Then, the size of the samples is small, and the participants come from a similar cultural background and working environment. It suggests that the more objective standard about the outstanding new nurses should be established, and some qualitative longitudinal studies can be carried out to further validate and improve the theoretical framework, which greatly increase the scientific nature of the theory and the guidance of the practice.

\section{Conclusion}

The resilience of the outstanding new nurses is a dynamic process with the mutual promotion between risk factors and protective factors. The successful transformation of the role does not mean the end of the development of nurses' resilience. The experience from the outstanding new nurses can help more new nurses go through the hardship of the career, and achieve role transformation. Nursing managers should understand the pressure and challenge of the transition period of the new nurses with the combination with the characteristics of the new nurse's growth stages, pay more attention to the improvement of the professional development and support system, and support the positive internal protection factors to enhance the development of the resilience of the new nurses.

\section{Conflict of Interest}

The authors declared no conflict of interest.

\section{Acknowledgements}

The research was supported by Jiangsu Modern Hospital Management Research Center (JSY-1-2016-068).

\section{References}

[1] Mcgowan, J. E., \& Murray, K. (2016). Exploring resilience in nursing and midwifery students: a literature review. Journal of Advanced Nursing, 72 (10), 2272-2283.

[2] Lu, H., Zhang, Y. Q., \& Wang, L. (2016). Research progress in nurses' mental resilience. Journal of Nursing Science, 31 (4), 93-96. In Chinese.

[3] Jewell, A. Supporting the novice nurse to fly: a literature review. Nurse Education in Practice, 2013, 13 (4), 323-327.

[4] Walker, A., Earl, C., Costa, B., \& Cuddihy, L. (2013). Graduate nurses' transition and integration into the workplace: a qualitative comparison of graduate nurses' and Nurse Unit Managers' perspectives. Nurse Education Today, 33 (3), 291-296.

[5] Al, A. H., Cooke, H., \& Pryjmachuk, S. (2015). The experiences of newly graduated nurses during their first year of practice in the Sultanate of Oman-A case study. International Journal of Nursing Studies, 52 (11), 1723-1734. 
[6] Liu, Y., Wu, L. M., Chou, P. L., Chen, M. H., Yang, L. C., \& Hsu, H. T. (2016). The Influence of Work-Related Fatigue, Work Conditions, and Personal Characteristics on Intent to Leave Among New Nurses. Journal of Nursing Scholarship, $48(1), 66-73$.

[7] Shi, R. F., Liu, Y. Y., \& Zhang, Z. L. (2016). The turnover status of nurse in tertiary hospitals of Guangdong province. Chinese Nursing Management, 16 (11), 1503-1506. In Chinese.

[8] Lavoie-Tremblay, M., Fernet, C., Laviqne, G. L., \& Austin, S. (2016). Transformational and abusive leadership practices: impacts on novice nurses, quality of care and intention to leave. Journal of Advanced Nursing, 72 (3), 582-592.

[9] Rondeau, K. V., \& Wagar, T. H. (2016). Human resource management practices and nursing turnover. Journal of Nursing Education \& Practice, 6 (10), 101-109.

[10] Li, Y., \& Jones, C. B. (2013). A literature review of nursing turnover costs. Journal of Nursing Management, 21 (3), 405-418.

[11] Rushton, C. H., Batcheller, J., Schroeder, K., \& Donohue, P. (2015). Burnout and Resilience Among Nurses Practicing in High-Intensity Settings. American Journal of Critical Care, 24 (5), 412-420.
[12] Hart, P. L., Brannan, J. D., \& De, C. M. (2014). Resilience in nurses: an integrative review. Journal of Nursing Management, $22(6), 720-34$.

[13] Blamire, L. (2014). Midwives' experiences of workplace resilience. Pract Midwife, 17 (10), 29-32.

[14] Reyes, A. T., Andrusyszyn, M. A., Iwasiw, C., Forchuk, C., \& Babenko-Mould, Y. (2015). Resilience in Nursing Education: An Integrative Review. Journal of Nursing Education, 54 (8), 438-444.

[15] Hatler, C., \& Sturgeon, P. (2013). Resilience Building: A Necessary Leadership Competence. Nurse Leader, 11 (4), 32-34.

[16] Zander, M., Hutton, A., \& King, L. (2013). Exploring resilience in paediatric oncology nursing staff. Collegian, 20 (1), 17-25.

[17] Cope, V., Jones, B., \& Hendricks, J. (2015). Why nurses chose to remain in the workforce: Portraits of resilience. Collegian, 23 (1), 87-96.

[18] Aburn, G., Gott, M., \& Hoare, K. What is resilience? An Integrative Review of the empirical literature. Journal of Advanced Nursing, 2016, 72 (5):980-1000. 